\title{
RINGS WITH UNIQUE ADDITION
}

\author{
R. E. JOHNSON
}

Dedicated to the Memory of Tibor Szele

Introduction. The ring $\{R ;+, \cdot\}$ is said to have unique addition if there exists no other ring $\left\{R ;+^{\prime}, \cdot\right\}$ having the same multiplicative semigroup $\{R ; \cdot\}$.

If $\{R ;+, \cdot\}$ and $\left\{R ;+^{\prime}, \cdot\right\}$ are different rings, then the 1-1 mapping $\theta: a \theta=a, a \in R$, of $\{R ;+, \cdot\}$ onto $\left\{R ;+^{\prime}, \cdot\right\}$ is multiplicative but not additive. Conversely, if there exists a 1-1 mapping $\theta$ of ring $\{R ;+, \cdot\}$ onto ring $\left\{S ;+^{\prime}, \cdot\right\}$ that is multiplicative but not additive, then the ring $R$ does not have unique addition. For we need only define $+^{\prime}$ on $R$ by: $a+^{\prime} b=\left(a \theta+^{\prime} b \theta\right) \theta^{-1}$ to obtain a new addition operation on $R$. Thus, it is clear that every 1-1 multiplicative mapping of ring $R$ onto some ring $S$ is additive if and only if $R$ has unique addition.

Rickart [1] has shown that a semi-simple ${ }^{1}$ ring satisfying certain minimum conditions has unique addition. We shall extend Rickart's results to a larger class of rings with minimum conditions in this paper. We have not been able to find any general results for rings without minimum conditions.

Preliminary remarks. If the multiplicative semigroup $\{R ; \cdot\}$ can be made into a ring, then there must exist a unique zero element 0 in $R$ such that $0 a=a 0=0$ for every $a \in R$. Let us assume that $R$ has a zero element 0 . An operation o on $R$ will be called a DO-operation if the following two conditions are satisfied:

(D) $(a \circ b) c=a c \circ b c, c(a \circ b)=c a \circ c b, a, b, c \in R$.

(O) $a \circ 0=0 \circ a=0, a \in R$.

If $a \circ b=0$ for every $a, b \in A$, a subset of $R$, then $\circ$ is said to vanish on $A$.

A ring without unique addition has DO-operations defined on it in an obvious way. Thus, if $\{R ;+, \cdot\}$ and $\left\{R ;+^{\prime}, \cdot\right\}$ are different rings, define $O$ on $R$ as follows:

$$
a \circ b=(a+b)-\left(a++^{\prime} b\right), \quad a, b \in R .
$$

It is easily verified that $\mathrm{O}$ is a DO-operation that does not vanish on $R$.

Presented to the Society December 29, 1956; received by the editors April 3, 1957.

1 While Rickart does not specifically say that the ring is semi-simple in his Theorem II, it is not difficult to show that his assumptions imply semi-simplicity. 
It is clear from our remarks above that if all DO-operations on a ring $R$ vanish on $R$, then $R$ has unique addition. This fact will play a primary role in proving the uniqueness of addition on the rings of the next section.

We shall designate by $A^{r}\left(A^{l}\right)$ the right (left) annihilator of the subset $A$ of a ring $R$. We shall also designate by $\mathscr{L}(R)$ the lattice of all right ideals of $R$ and by $\mathscr{L}^{\mathbf{\Lambda}}(R)$ the sublattice containing all $A \in \mathfrak{L}(R)$ for which $A \cap B \neq 0$ for every nonzero $B \in \mathfrak{L}(R)$.

Rings with zero singular ideal. If $R$ is a ring, the set

$$
R^{\mathbf{\Lambda}}=\left\{x ; x \in R, x^{r} \in \mathfrak{L}^{\mathbf{\Delta}}(R)\right\}
$$

is an ideal of $R$, called the singular ideal in [2]. We shall assume in the remainder of the paper that $R$ is a ring such that

$$
R^{\boldsymbol{\Lambda}}=0 \text {. }
$$

For each $A \in \mathfrak{L}(R)$, let us define

$$
A^{8}=\left\{x ; x \in R, x^{-1} A \in \mathfrak{L}^{\mathbf{\Delta}}(R)\right\},
$$

where $x^{-1} A=\{y ; x y \in A\}$. It is easily shown that $A^{s} \in \mathcal{L}(R)$, and that $s$ is a closure operation on $\mathscr{L}(R)[3, \S 6]$.

Lemma 1. If $A \in \mathfrak{L}(R)$ and $\circ$ is a DO-operation on the ring $R$ that vanishes on $A$, then $\circ$ vanishes on $A^{s}$.

Proof. Let $x, y \in A^{s}$ and $B=x^{-1} A \cap y^{-1} A$, an element of $\mathscr{L}^{\mathbf{\Lambda}}(R)$. Since $x B \subset A$ and $y B \subset A,(x \circ y) B=0$; and therefore $x \circ y=0$ since $(x \circ y)^{r} \in \mathcal{L}^{\Delta}(R)$. This proves the lemma.

A minimal nonzero element of $\mathscr{L}^{\varepsilon}(R)=\left\{A^{s} ; A \in \mathfrak{L}(R)\right\}$, if such exists, is called an atom. The closure operation $s$ on $\mathscr{L}(R)$ is called atomic if each nonzero element of $\mathscr{L}^{s}(R)$ contains at least one atom. The union $S$ of the atoms of $\mathscr{L}^{s}(R)$ is called the base of $R$. It may be shown that $S$ is an ideal of $R$ and that $S^{l}=0$ if and only if $s$ is atomic.

If $R$ is a semi-simple ring with minimal right ideals and if the union $S$ of the minimal right ideals of $R$ is such that $S^{l}=0$, then clearly $R^{\mathbf{\Lambda}}=0$ and $s$ is an atomic closure operation on $\mathfrak{L}(R)$. An example of a ring that satisfies our assumptions but is not semi-simple is given below.

EXAMPLE 1. Let $I$ be the ring of integers and $R=e_{11} I+e_{21} I+e_{22} I$, the ring of $2 \times 2$ triangular matrices over $I$. This ring has a radical, namely the nilpotent ideal $e_{21} I$. Each $A \in \mathscr{L}^{\mathbf{\Delta}}(R)$ necessarily contains elements of the form $k_{1} e_{11}+k_{2} e_{21}$ with $k_{i} \neq 0$. Clearly, then, $R^{\mathbf{\Lambda}}=0$. The right ideals $e_{11} R$ and $e_{22} R$ are atoms of $\mathscr{L}^{s}(R)$. Hence $s$ is atomic even though the ring $R$ itself has no minimal right ideals. 
If $s$ is atomic, it is known $[3,6.9]$ that for $x \in R,(x R)^{s}$ is an atom of $\mathfrak{L}^{s}(R)$ if and only if $x^{r}$ is a maximal element $(\neq R)$ of $\mathscr{L}^{s}(R)$.

Lemma 2. If $x, y \in R$ are chosen so that $x^{r}+y^{r} \in \mathcal{L}^{\mathbf{\Delta}}(R)$, then $x \circ y=0$ for every DO-operation o on $R$.

Proof. Since $(x \circ y) x^{r}=(x \circ y) y^{r}=0,(x \circ y)\left(x^{r}+y^{r}\right)=0$ and $x \circ y$ $=0$.

Lemma 3. If $s$ is atomic and $A$ is an atom of $\mathscr{L}^{s}(R)$ such that $A^{r}$ is not a maximal element of $\mathcal{L}^{*}(R)$, then every $D O$-operation vanishes on $A$.

Proof. Let $\circ$ be a DO-operation on $R$. If $x$ and $y$ are nonzero elements of $A$ such that $x^{r} \neq y^{r}$, then $x^{r}+y^{r} \in \mathcal{L}^{\Delta}(R)$ and $x \circ y=0$ by Lemma 2. If $x^{r}=y^{r}$, there must exist some nonzero $z \in A$ such that $z^{r} \not x^{r}$, for otherwise $A^{r}=x^{r}$, a maximal element of $\mathfrak{L}^{s}(R)$. Now $(y+z)^{r}$ is a maximal element of $\mathfrak{L}^{s}(R)$ and $(y+z)^{r} \neq x^{r}$; hence $x \circ(y+z)=0$. Since $[x \circ(y+z)] z^{r}=(x \circ y) z^{r}=0$ and $(x \circ y) x^{r}=0,(x \circ y)\left(x^{r}+z^{r}\right)=0$ and $x \circ y=0$. This proves the lemma.

The reason for the hypothesis that $A^{r}$ is not maximal in $\mathscr{L}^{s}(R)$ is apparent if we let $R$ be a field. Then $R$ is an atom of $\mathscr{L}^{s}(R)$ and $R^{r}=0$, a maximal element of $\mathfrak{L}^{s}(R)$. However, not all fields have unique addition, as Rickart shows in his paper.

Lemma 4. If $s$ is atomic and $A \in \mathscr{L}^{s}(R)$ is an atom such that $A^{r}$ is maximal in $\mathscr{L}^{s}(R)$, then there exists an atom $B \in \mathcal{L}^{s}(R)$ such that $B^{r}=A^{r}$ and $B$ is an integral domain.

Proof. Since $R^{\boldsymbol{\Lambda}}=0$, there exists an atom $B \in \mathcal{L}^{s}(R)$ such that $B \cap A^{r}=0$. Thus $x b \neq 0$ and $x b b^{\prime} \neq 0$ for each nonzero $x \in A$ and $b, b^{\prime} \in B$. Hence $B$ is an integral domain, with $B^{r}=A^{r}$.

Let us call two atoms $A$ and $B$ of $\mathscr{L}^{s}(R)$ perspective $[3, \S 6]$, and write $A \sim B$, if and only if $a^{r}=b^{r}$ for some nonzero $a \in A$ and $b \in B$. If $R$ is semi-simple, two minimal right ideals are perspective if and only if they are isomorphic as right $R$-modules. We shall also call an atom $A$ of $\mathscr{L}^{s}(R)$ isolated if $B^{r}$ is a maximal element of $\mathfrak{L}^{s}(R)$ for every $B \sim A$.

Lemma 5. If $s$ is atomic and $\circ$ is a DO-operation on the ring $R$, then o vanishes on each nonisolated atom of $\mathfrak{L}^{s}(R)$.

Proof. Let $A$ be a nonisolated atom of $\mathscr{L}^{s}(R)$. If $A^{r}$ is not maximal in $\mathscr{L}^{s}(R)$, $\circ$ vanishes on $A$ by Lemma 3. If $A^{r}$ is maximal, then by Lemma 4 there exists an atom $B$ such that $B^{r}=A^{r}$ and $B$ is an integral domain. Since $B$ is nonisolated, there exists an atom $C \sim A$ such that $C^{r}$ is not maximal in $\mathfrak{L}^{8}(R)$. Also $C \sim B$ and there exist nonzero 
$b \in B$ and $c \in C$ such that $b^{r}=c^{r}$. Clearly $b B \neq 0$, and therefore $c B \neq 0$. Since $\circ$ vanishes on $C, c(x \circ y)=c x \circ c y=0$ for every $x, y \in B$. Hence $b(x \circ y)=b x \circ b y=0$ for every $x, y \in B$, and $\circ$ vanishes on $b B$, and also on $B=(b B)^{s}$ by Lemma 1 .

Now for any nonzero $a \in A, a B \neq 0$. Since $a(x \circ y)=0$ for every $x, y \in B$, o vanishes on $a B$ and also on $A=(a B)^{s}$. This proves the lemma.

We might suspect from Lemma 5 that if the ring $R$ has no isolated atoms, so that every DO-operation vanishes on each atom, then the ring has unique addition. That this is not true is illustrated by the following example.

ExAmple 2. Let $F$ be the field of integers modulo 2 and $R=e_{11} F$ $+e_{21} F+e_{31} F+e_{32} F$ be a subring of the ring of $3 \times 3$ matrices over $F$. Define the mapping $\theta$ of $R$ onto $R$ as follows:

$$
\text { if } a=\sum_{i} \alpha_{i} e_{i 1}+\alpha e_{32}, \quad \text { let } a \theta=a+\alpha_{1} \alpha_{2} e_{31} \text {. }
$$

It is an easy exercise to prove that $\theta$ is a $1-1$ multiplicative mapping of $R$ onto $R$ and that $\theta^{2}$ is the identity mapping. Since

$$
\left(e_{11}+e_{21}\right) \theta=e_{11}+e_{21}+e_{31}, \quad e_{11} \theta+e_{21} \theta=e_{11}+e_{21},
$$

clearly $\theta$ is not additive on $R$. If

$$
b=\sum_{i} \beta_{i} e_{i 1}+\beta e_{32}
$$

then $R$ has another addition $t^{\prime}$ defined by:

$$
a+{ }^{\prime} b=(a \theta+b \theta) \theta=a+b+\left(\alpha_{1} \beta_{2}+\alpha_{2} \beta_{1}\right) e_{31} .
$$

All the atoms of $\mathscr{L}^{s}(R)$ for this example are perspective, and one of them, namely $e_{31} F+e_{32} F$, has an annihilator $e_{31} F+e_{32} F$ which is not maximal in $\mathscr{L}^{s}(R)$. Thus every DO-operation 0 vanishes on each atom of $\mathcal{L}^{s}(R)$ by Lemma 5 , although o does not necessarily vanish on $R$, since $R$ does not have unique addition.

It is clear from this example that some further restriction must be placed on the ring $R$ to insure unique addition. We shall give two possible ways of doing this.

THEOREM 1. If $s$ is atomic and the base $S$ of $R$ is such that $S^{r}=0$, and if $\mathscr{L}^{s}(R)$ has no isolated atoms, then the ring $R$ has unique addition.

Proof. Let o be a DO-operation on $R$. By Lemma 5, o vanishes on each atom of $\mathfrak{L}^{s}(R)$. If $x, y \in R$, then $c(x \circ y)=c x \circ c y=0$ for every $c$ in some atom $C$. Since $S^{r}=0$, evidently $x \circ y=0$. This proves the theorem. 
The ring $R$ of Example 1 satisfies the conditions of Theorem 1 . Therefore addition is unique for this ring. In Example 2, $S=R$ and $S^{r}=e_{31} F+e_{32} F \not 10$.

TheOREM 2. If $s$ is atomic and, for each atom $A$ of $\mathfrak{L}^{s}(R), A^{r}$ is not maximal in $\mathfrak{L}^{\mathbb{s}}(R)$, then the ring $R$ has unique addition.

Proof. Let $\circ$ be a DO-operation on $R$. If $A$ and $B$ are atoms and $x \in A, y \in B$, then $x \circ y=0$ by Lemma 2 if $x^{r} \neq y^{r}$. If $x^{r}=y^{r} \neq R$, we may select a nonzero $z \in A$ such that $z^{r} \neq x^{r}$. Since $(x+z)^{r} \neq y^{r}$, $(x+z) \circ y=0$ by Lemma 2. Hence $[(x+z) \circ y] z^{r}=(x \circ y) z^{r}=0$, $(x \circ y) x^{r}=0$, and $x \circ y=0$. We conclude that $x \circ y=0$ if $x$ and $y$ are in atoms of $\mathscr{L}^{8}(R)$.

If $x, y \in R$, then for every $a \in A$, an atom of $\mathfrak{L}^{s}(R)$, either $x a=0$ or $(x a)^{r}$ is a maximal element of $\mathscr{L}^{s}(R)$, and similarly for $y a$. Hence each of $x a$ and $y a$ is in an atom of $\mathscr{L}^{s}(R)$, and $(x \circ y) a=x a \circ y a=0$ by the previous paragraph. Thus $(x \circ y) S=0$ and $x \circ y=0$ since $S^{l}=0$. This proves the theorem.

The ring $R$ of Example 2 fails to satisfy the conditions of Theorem 2 in that many of the atoms have maximal annihilators.

\section{BIBLIOGRAPHY}

1. C. E. Rickart, One-to-one mappings of rings and lattices, Bull. Amer. Math. Soc. vol. 54 (1948) pp. 758-764.

2. R. E. Johnson, The extended centralizer of a ring over a module, Proc. Amer. Math. Soc. vol. 2 (1951) pp. 891-895.

3. - - Structure theory of faithful rings, II. Restricted rings, Trans. Amer. Math. Soc. vol. 84 (1957) pp. 523-544.

Smith College 